\title{
Seismic Tomography of the Near Solar Surface
}

\author{
L. Gizon ${ }^{1}$, T. L. Duvall Jr² \& R. M. Larsen ${ }^{1,3}$ \\ ${ }^{1}$ HEPL, Stanford University, Stanford CA 94305, USA. \\ ${ }^{2}$ NASA/Goddard Space Flight Center, Greenbelt MD 20771, USA. \\ ${ }^{3}$ SCCM, Stanford University, Stanford CA 94305, USA. \\ e-mail: Igizon@solar.Stanford.edu
}

\begin{abstract}
Surface gravity waves have been used to probe flows in the two megameters beneath the photosphere using the techniques of timedistance helioseismology. The results suggest that supergranule velocities are smaller than at the surface. The outward flow outside a sunspot penumbra (the moat) is observed, as is an inward flow in the region beyond the moat.
\end{abstract}

Key words. Helioseismology—supergranulation—sunspots.

\section{Time-distance helioseismology with surface gravity waves}

Local helioseismology diagnostics open the prospect of mapping the three-dimensional structure and dynamics of the upper solar convection zone. Duvall \& Gizon (2000) recently applied time-distance helioseismology to surface gravity waves, to probe horizontal flows in the first two megameters below the photosphere. Promising results were obtained using a simple calibration instead of a proper inversion.

Here we have analyzed an 8-hour long time series of dopplergrams observed on 1998 December 6 in the MDI high-resolution field of view (Scherrer et al. 1995). Rotation was removed. The datacube was Fourier transformed in time and space, and acoustic waves filtered out. The temporal cross correlations were computed and averaged using the quadrant geometry, as described by Duvall \& Gizon (2000). For each pixel location, $\mathrm{x}$, we obtained three cross-correlation functions, denoted $C^{\mathrm{EW}}$, $C^{\mathrm{SN}}$ and $C^{\mathrm{IO}}$, which depend on correlation time $\mathrm{T}$ and distance $\Delta$ from $\mathrm{x}$. They respectively contain local information about the westward component, northward component, and divergence of the flow. A fourth cross-correlation function, $\mathrm{C}^{0}$, was constructed by averaging over all possible pairs of points; it is symmetric with respect to correlation time. Flows introduce a correlation-time asymmetry in $C^{\mathrm{EW}}, C^{\mathrm{SN}}$ and $C^{\mathrm{I}}$, which we measure by cross correlation with the template $\mathrm{C}^{0}$. We finally get time-asymmetry maps $\delta \tau^{\mathrm{EW}}, \delta \tau^{\mathrm{SN}}$, and $\delta \tau^{\mathrm{IO}}$ for each distance $\Delta$. Further averaging over $\Delta$ in the range $4.5-11.9 \mathrm{Mm}$ provides us with three maps, labeled $\overline{\delta \tau}{ }^{\mathrm{EW}}, \overline{\delta \tau}$ and $\overrightarrow{\delta \tau}$, which are functions of $\mathrm{x}$. 

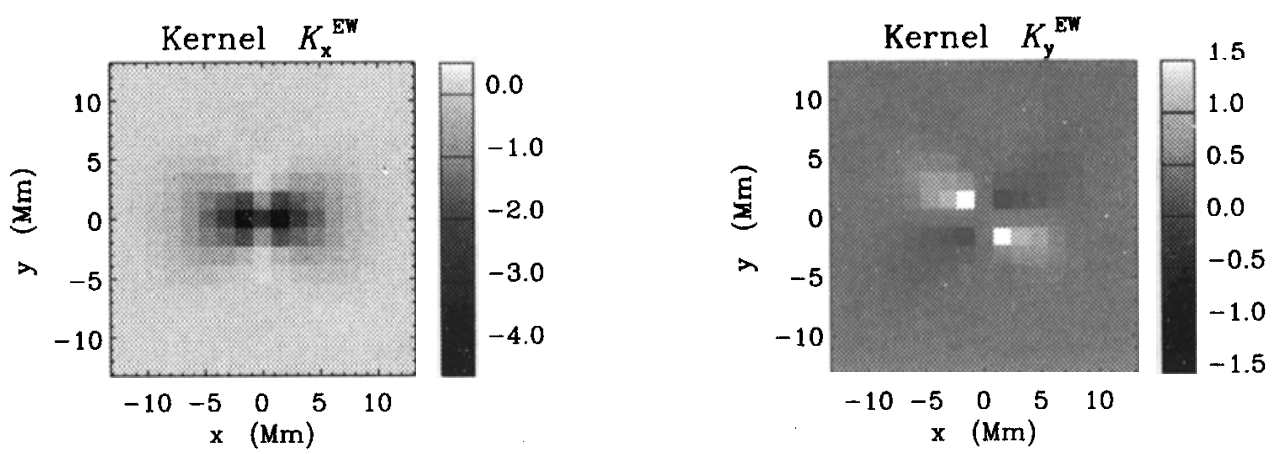

Figure 1. Kernels $K_{x}^{\mathrm{EW}}$ and $K_{y}^{\mathrm{EW}}$ which provide a relationship between the time-asymmetry map $\bar{\delta}^{\mathrm{EW}}$ and the horizontal flow $\mathrm{U}$. The kernels were averaged down to a resolution of $1.64 \mathrm{Mm}$. They are expressed in units of $10^{-3} \mathrm{~s}^{2} \mathrm{~m}^{-1}$ per pixel.

\section{2-D sensitivity kernels for horizontal flows}

It is not appropriate to employ ray theory to solve the forward problem, because the flow can vary on a horizontal scale smaller than the wavelength, which is $\sim 5 \mathrm{Mm}$ for a $3-\mathrm{mHz}$ surface gravity wave. Instead, we calculated the wavefield in the first Born approximation (see Woodward 1992). We restricted our attention to a simplified problem, in which the prescribed flow (U) is steady, horizontal, and depth independent. Furthermore the random sources of oscillations are assumed to be of pressure type, uniformly distributed on the surface, and spatially uncorrelated (Woodard 1997). Realistic frequency-dependent damping is included. Dispersion is built in. Under these conditions the scattered wavefield can be expressed as a 2-D surface integral. We can then relate time-asymmetry measurements (as defined above) to the two horizontal components of the flow following the procedure described in Tong et al. (1998). We write $\bar{\delta}^{\mathrm{i}}=K_{x}^{l} * U_{x}+K_{y}^{l} * U_{y}$ for $i \in\{\mathrm{EW}, \mathrm{SN}, \mathrm{IO}\}$, where '*'denotes 2-D convolution. The kernels $K_{x}^{\mathrm{EW}}$ and $K_{y}^{\mathrm{EW}}$ are shown in Fig. 1. Wave effects are smeared out by the spatial averaging over quadrants and distances.

\section{Iterative deconvolution}

To obtain the horizontal components $U_{x}$ and $U_{y}$ of the flow we solve the overdetermined system of equations mentioned above in the least squares sense. We apply the iterative LSQR algorithm and use the 2-D FFT to compute the convolutions involved. A regularized solution with small $l_{2}$-norm is obtained by stopping LSQR after a small number of iterations, $k$.

The inversion procedure was tested on artificial data corresponding to a hexagonal convective flow pattern. For moderate (10-100) signal-to-noise levels, the values of $k$ found by the L-curve criterion or by Monte-Carlo generalized cross validation (Hansen 1998) are close to optimal and result in very accurate recovery of the artificial velocity signals. For the real observations, which are noisier, this is not possible, and instead we choose $k=2$ based on inversions of artificial data with low signal-to noise. 


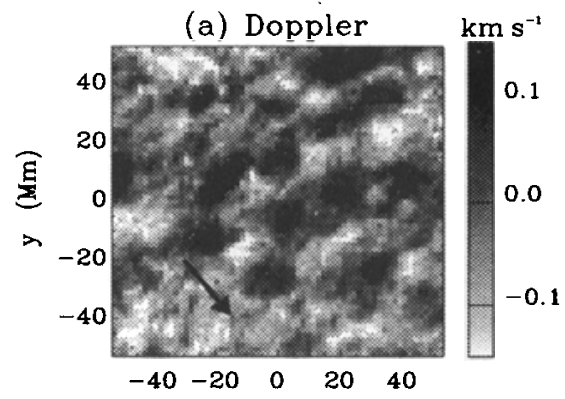

(b) Line-of-sight $\mathrm{km} \mathrm{s}^{-1}$

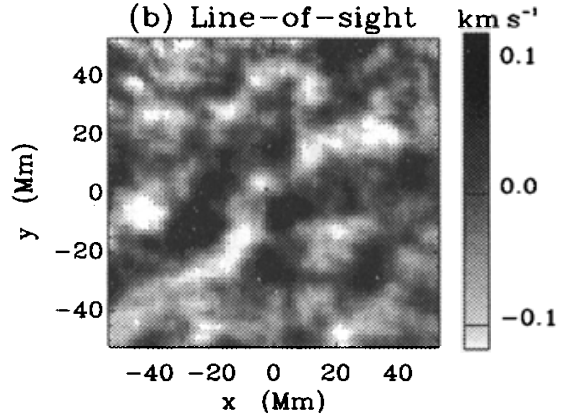

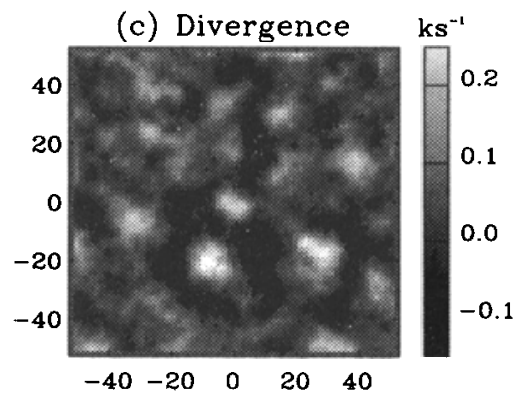

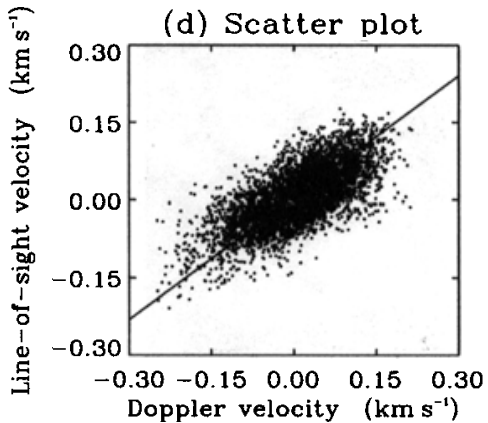

Figure 2. (a) 8-hour averaged dopplergram. The arrow points towards disk center. (b) Lineof-sight projection of the inferred horizontal velocity field obtained by deconvolution. (c) Horizontal divergence of the inferred flow. (d) Scatter plot of (b) versus (a); the straight line with slope 0.8 is a fit assuming equal errors in both coordinates. All maps have a spatial resolution of $1.64 \mathrm{Mm}$.

\section{Results}

Fig. 2 displays inversions obtained for a relatively quiet region. The supergranulation cells are easily identified in the divergence map. Also shown is the line-of-sight component of the inferred flow. The correlation with the average dopplergram is high (correlation coefficient 0.7). It appears that there is less high spatial frequency signal in our image. Perhaps this is a consequence of measuring a flow averaged over $2 \mathrm{Mm}$ in depth. We also find the inferred flow to be weaker than the surface flow by a factor of 0.8. Supergranular motion may indeed depend on depth since density varies very rapidly in the near surface layers.

Results for a region including a sunspot are shown in Fig. 3. We detect a radial outflow from the sunspot center extending out to $30 \mathrm{Mm}$, with values up to $1 \mathrm{~km} \mathrm{~s}^{-1}$. This is a manifestation of the moat flow. The moat is surrounded by a counter flow, suggestive of a downflow at the moat boundary. In the penumbra, the inferred outflow is significantly smaller than the Evershed flow observed in the Doppler image. The Evershed flow may thus be very shallow. It is not straightforward to reconcile the present findings with previous observations of downflows below sunspots (Duvall et al. 1996). We caution that spatially varying damping could introduce an additional time-asymmetry component (Woodard 1997) which is not accounted for in this study. 


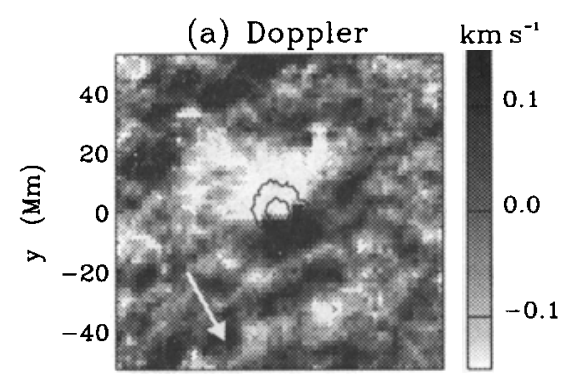

(b) Line-of-sight $\mathrm{km} \mathrm{s}^{-1}$

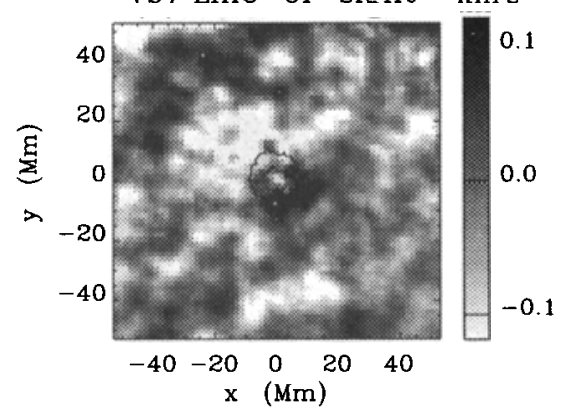

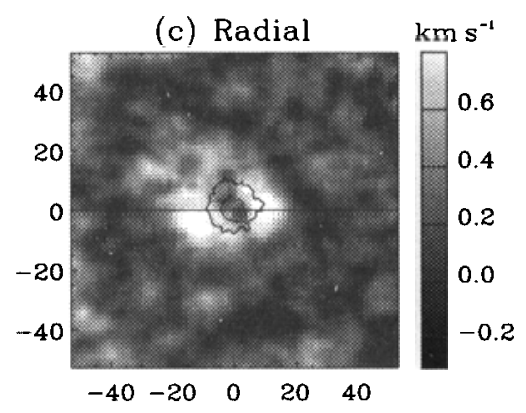

(d) Cut $y=0$

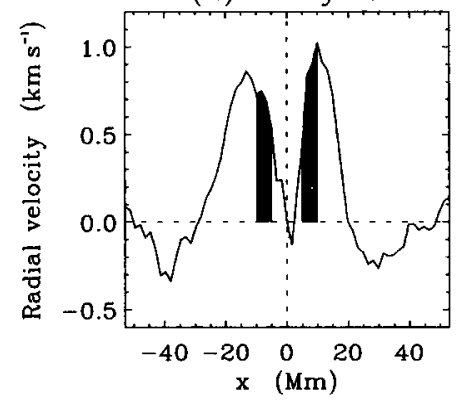

Figure 3. (a) 8-hour averaged dopplergram. The contour lines mark the boundaries of the umbra and penumbra. Doppler velocities vary between -0.8 and $0.3 \mathrm{kms}^{-1}$ in the penumbra. (b) Line-of-sight projection of the inferred horizontal velocity field. The correlation coefficient between (a) and (b) is 0.6. (c) Radial component of inferred flow measured from sunspot center. (d) Cut at $y=0$ through (c); the shaded regions indicate the location of the penumbra.

This work was supported by NASA grant NAG5-3077. SOHO is a mission of cooperation between ESA and NASA. We thank Phil Scherrer for discussions.

\section{References}

Duvall, T. L. Jr et al. 1996, Nature, 379, 235.

Duvall, T. L. Jr, Gizon, L. 2000, Solar Phys., 192, 177.

Hansen, P. C. 1998, Rank-Deficient and Discrete III-Posed Problems: Numerical Aspects of Linear Inversion, SIAM, (Philadelphia).

Scherrer, P. H. et al. 1995, Solar Phys., 162, 129.

Tong, J. et al. 1998, Geophys. Res. Lett., 25, 1983.

Woodard, M. F. 1997, Astrophys. J., 485, 890.

Woodward, M. J. 1992, Geophys., 57, 15. 